\section{RMD Open}

Rheumatic \&

Musculoskeletal Diseases

\title{
Parenting problems postpartum can be detected early in pregnancy in patients with rheumatoid arthritis
}

Hieronymus T W Smeele (D), Yael A de Man, Esther Röder, Hetty Wintjes, J M W Hazes, Radboud J E M Dolhain

To cite: Smeele HTW, de Man YA, Röder E, et al. Parenting problems postpartum can be detected early in pregnancy in patients with rheumatoid arthritis. RMD Open 2020;6: e001276. doi:10.1136/ rmdopen-2020-001276

- Additional material is published online only. To view please visit the journal online (http://dx.doi.org/10.1136/rmdo pen-2020-001276)

Received 17 April 2020 Revised 3 June 2020 Accepted 17 June 2020

\section{Check for updates}

(C) Author(s) (or their employer(s)) 2020. Re-use permitted under CC BY-NC. No commercial re-use. See rights and permissions. Published by BMJ.

Rheumatology, Erasmus MC, Rotterdam, Netherlands

Correspondence to Hieronymus T W Smeele; h.smeele@erasmusmc.nl

\section{ABSTRACT}

Objective To describe parenting disability postpartum in patients with rheumatoid arthritis (RA) using the Parenting Disability Index and to determine early in pregnancy which patients will face parenting problems postpartum.

Methods Data were collected from a prospective study on pregnancy and RA (Pregnancy induced Amelioration of Rheumatoid Arthritis study). Postpartum visits were performed at 6, 12 and 26 weeks after delivery. Domains causing parenting difficulties were identified. A multivariate logistic regression model to identify which patients develop parenting disabilities postpartum with patient characteristics in the first trimester as covariates was performed.

Results 148 patients were eligible for this study. The domains carrying, hygiene, feeding, getting up and down, and household/shopping were frequently scored as difficult. Maintaining discipline, taking care of the children when sick, listening and having other children over caused the least problems. $30.1 \%$ of patients with RA report low parenting disability, $30.9 \%$ reports intermediate disability and $39.0 \%$ reports high disability. Patients with a low Health Assessment Questionnaire (HAQ)-score in the first trimester (OR 9.2, 95\% $\mathrm{Cl} 3.0$ to 27.7, $\mathrm{p}<0.001$ ) and low disease activity in the first trimester (Disease Activity Score 28 -joint count $C$ reactive protein<3.2) (OR 4.8, 95\% Cl 1.8 to $12.9, \mathrm{p}=0.002)$ were likely to report low parenting disability postpartum. Patients with a longer disease duration ( $0 \mathrm{R} 0.87,95 \% \mathrm{Cl} 0.79$ to 0.95 , $p=0.003$ ) were less likely to report low parenting disability postpartum. A high HAQ-score in the first trimester (OR 4.54, $95 \% \mathrm{Cl} 1.99$ to $10.34, \mathrm{p}<0.001)$ and erosive disease (OR $2.32,95 \% \mathrm{Cl} 1.00$ to $5.35, \mathrm{p}=0.049$ ) increased the risk of high parenting disability postpartum.

Conclusion Physical domains of parenting postpartum are most commonly affected in patients with $\mathrm{RA}$. When counselling patients with RA, a HAQ-score in the first trimester is the most reliable marker to identify patients that develop parenting disability after delivery.

\section{INTRODUCTION}

One of the most frequent questions of patients with rheumatoid arthritis (RA) during counselling sessions is, 'does my disease influence my ability to take care of my children?' Women

\section{Key messages}

What is already known about this subject?

- Women with RA are concerned about their parenting capacities after giving birth and experience an unmet need for information and focused care.

What does this study add?

- Almost $40 \%$ of mothers with RA reports high parenting disability postpartum.

- Physical domains are more often affected than social and psychosocial domains in mothers with RA.

- $\mathrm{HAQ}$ scores in the first trimester, disease activity in the first trimester, disease duration and the presence of erosive disease are predictive for developing parenting disability after delivery.

How might this impact on clinical practice?

- When counselling patients with RA early in pregnancy, disease activity, disease duration, the presence of erosive disease and especially $\mathrm{HAQ}$ scores can help identifying patients at risk for developing parenting problems.

with RA are concerned about their parenting capacities after giving birth, with perceived inability to fulfil parenting roles. Previous studies identified feelings of guilt, anger and depression in mothers with RA. ${ }^{1}$ Although these problems were identified more than 20 years ago, recent studies still find an unmet need for information and focused care in mothers with a chronic rheumatic disease. ${ }^{23}$

Counselling patients with a wish to conceive is important to ensure safe pregnancy outcomes for mother and child. ${ }^{4}$ Parenting ability should be one of the topics discussed during these counselling sessions. Early identification of patients that will develop parenting problems gives these patients time to take additional precautions.

The Parenting Disability Index (PDI) was developed to measure parenting function and disability in women with a chronic rheumatic disease. ${ }^{5}$ It was developed and validated 
in a cohort with RA patients from the USA. Although this study included few mothers with young children (age $0-5$ years), more than half of these patients reported difficulty regarding the physical activity domains of parenting. This suggests that a majority of women with RA experience difficulties in parenting.

The goals of this study are to describe the affected domains of the PDI for young children ( $0-5$ years of age) over a 6 months postpartum period in a large sample of mothers with RA and to identify patients early in pregnancy who are at risk for developing parenting disabilities postpartum. The information provided in our study will help physicians and healthcare professionals to improve counselling on parenting abilities and to identify those patients who need extra care. Both will help women with RA better prepare for potential parenting challenges after delivery.

\section{METHODS}

\section{Patients}

Patients were derived from the Pregnancy-induced Amelioration of Rheumatoid Arthritis (PARA) cohort (2002-2010). ${ }^{6}$ The PARA cohort is a large, prospective cohort study on RA and pregnancy from the Netherlands. Patients included in this study fulfilled the 1987 American College of Rheumatology for RA criteria. ${ }^{7}$ The PARA study was approved by the Erasmus MC ethics review board and was executed in compliance with the Helsinki Declaration. All patients gave a written informed consent.

\section{Data collection}

In the PARA study, visits were performed (preferably) before pregnancy, each trimester and three times postpartum $(6,12$ and 26 weeks). At all visits during pregnancy and all visits postpartum, participants filled in questionnaires, including the $\mathrm{PDI}^{5}$ and Health Assessment Questionnaire (HAQ). The HAQ measures the difficulty in performing activities of daily living in eight categories. ${ }^{8}$ Data on patient and disease characteristics were collected at baseline. From August 2004 onwards, all participants of the PARA study were asked to fill in PDI questionnaires postpartum.

\section{Parenting Disability Index}

The PDI is an extensive, validated questionnaire with 25 domains to quantify parenting disability in mothers with arthritis. ${ }^{5}$ This questionnaire for women with children between 0 and 5 (including five additional questions) was translated into Dutch by two translators. It was translated back into English by two native English-speaking translators. Consensus on the translated version was reached after two versions. All questions could be retained as in the English version. The translated Dutch questionnaire for children between 0 and 5 years is available in online supplementary A. The PDI uses a five-point response scale: $0=$ no difficulty, $1=$ some difficulty, $2=\mathrm{a}$ lot of difficulty, $3=$ unable to perform, $4=$ does not apply. The PDI has an additional category in which participants could state whether the task was performed less because of reasons related to arthritis. For all domains, except 'safety' and 'getting down to and up from the floor' domains, this additional question was asked. In the modified Parenting Disability score (mPDI), the domain score was increased to a 2 if a subject responded that she did an activity less due to arthritis. We calculated the mPDI score as described in literature. ${ }^{5}$

\section{Statistics}

Descriptive statistics are presented as numbers (n) and percentages $(\%)$. Values are given as mean \pm SD, number (percentage) or median \pm IQR. Records with available data in the first trimester of pregnancy and at least one time-point postpartum were included in the current analysis (complete case analysis).

Domains in which mothers with RA experienced parenting difficulty were described using descriptive statistics. We stratified patients to different parenting disability states: low parenting disability ( $0-4$ domains affected), medium parenting disability (5-12 domains affected) and high parenting disability (13-25 domains affected). Affected domains of parenting were visualised for the entire group of patients and for the different parenting disability states by using histograms.

Agreement in parenting disability states at the different visits postpartum was determined by kappa statistics. Categories for kappa values were in line with previous literature. ${ }^{9}$ Correlations between the calculated mPDI scores at 6,12 and 26 weeks postpartum were determined using Pearson's correlation coefficients. Correlation coefficients were interpreted as described in literature. ${ }^{10}$

To identify patients who are at risk for developing parenting disability, univariate logistic regression models were built for the analyses of patient characteristics associated with low parenting disability (low vs medium and high combined) and high parenting disability (high vs low and medium combined). Stepwise forward selection of covariates with a $\mathrm{p}$ value less than 0.2 in the univariate analysis was performed to create a multivariate model. $\mathrm{P}$ values $\leq 0.05$ in the final multivariate model were considered statistically significant. The following patient characteristics, obtained in the first trimester of pregnancy, were considered as covariates: age (years), disease duration (years), already having children (yes/no), socioeconomic status (years of education), erosive disease (yes/no), disease activity (Disease Activity Score (DAS) with three variables: 28 swollen and tender joint count and C reactive protein (CRP) (DAS28CRP)) and the HAQ score. For clinical purposes, we stratified disease activity to low or high (low disease activity DAS28CRP $<3.2$ ) and the HAQ score to low or high based on the median. All analyses were performed using STATA 15.

\section{RESULTS}

A detailed description of the 148 patients within the PARA cohort who were eligible for the current study is given in table 1. A total of 149 children were born in the current study, the mean maternal age was 33.2 years and 
Table 1 Clinical and demographic features from patients within the PARA cohort $(n=148)$ with rheumatoid arthritis

\begin{tabular}{ll}
\hline Mean age, years (SD) & $33.2(3.5)$ \\
\hline Median gestational age, weeks (IQR) & $39(38-40)$ \\
\hline Mean disease duration, years (SD) & $6.4(5.8)$ \\
\hline Rheumatoid factor positive, $\mathrm{n}(\%)$ & $106(71.6)$ \\
\hline ACPA positive, $\mathrm{n}(\%)$ & $92(62.2)$ \\
\hline Nulliparity, $\mathrm{n}(\%)$ & $73(49.3)$ \\
\hline Erosions, $\mathrm{n}(\%)$ & $89(60.1)$ \\
Smoking, $\mathrm{n}(\%)$ & $13(8.8)$ \\
\hline Education, years (SD) & $15.5(2.9)$ \\
Medication use postpartum (any use, $\mathrm{n}(\%)):$ & \\
- Prednisone & $61(41.2)$ \\
- Methotrexate & $54(36.5)$ \\
- Sulfasalazine & $49(33.1)$ \\
- Biological agents* & $20(13.5)$ \\
- Hydroxychloroquine & $18(12.2)$ \\
- Azathioprine & $2(1.4)$ \\
- Leflunomide & $1(0.7)$ \\
\hline
\end{tabular}

*adalimumab $n=8$, etanercept $n=13$.

ACPA, anti-citrullinated protein antibody; PARA, Pregnancyinduced Amelioration of Rheumatoid Arthritis.

the median gestational age of the newborn was 39 weeks. Of the 148 patients, $50.7 \%$ already had children, $71.6 \%$ were rheumatoid factor positive and $62.2 \%$ were anticitrullinated protein antibody positive; the mean duration of RA was 6.4 years. The average number of years of schooling was 15.5 (2.9 SD), indicating that participants finished at least a vocational education and training or started and/or finished a higher education.

The total number of available patients for analysis are as follows: at 6 weeks postpartum, $\mathrm{n}=116$; 12 weeks postpartum, $\mathrm{n}=123$; and 26 weeks postpartum, $\mathrm{n}=115$.

\section{Parenting postpartum}

Complete responses on all questions of the PDI, at 6, 12 and 26 weeks postpartum are shown in online supplemen tary tables $1-3$, respectively. The domains in which respondents had the most difficulty were picking up or carrying your child, doing household chores or shopping, taking your child out in the car, getting up and down to the floor to play your with child, taking care of your child's hygiene, feeding your child and using a car seat. The domains in which mothers with RA had the least difficulties were taking care of your child when he or she is sick, maintaining discipline with your child, having other children in your home and having the energy to be available to listen and talk with your child.

In this study, $78.5 \%$ of the patients reported any parenting problems at 6 weeks postpartum, $86.2 \%$ reported any problems at 12 weeks postpartum and $86.1 \%$ at 26 weeks postpartum. Twelve weeks postpartum was the visit with the highest percentage of any parenting difficulty reported. At this visit, low, medium and high parenting disability were observed in $37(30.1 \%), 38(30.9 \%)$ and $48(39.0 \%)$ of the patients, respectively. Figure 1 shows the percentage of patients reporting any parenting difficulty per domain at 12 weeks postpartum; this is displayed for the entire group and displayed after stratification for parenting disability state. The most commonly reported domains in the low parenting disability group were getting up and down to the floor to play with your child $(24.3 \%)$ and getting up with your child (18.9\%). In the medium parenting disability group, the domainspicking up or carrying your child $(86.8 \%)$ and doing household chores or shopping $(73.7 \%)$ were most frequently reported. In the high parenting disability group, the domains picking up or carrying your child (100\%), doing household chores or shopping $(100 \%)$, getting up and down to the floor to play with your child $(97.7 \%)$ and taking care of your child's hygiene $(91.7 \%)$ were most frequently reported.

The calculated mPDI scores did not change over time: 6 weeks postpartum mean mPDI $0.66(0.57 \mathrm{SD})$ at 6 weeks postpartum, mean mPDI $0.67(0.56 \mathrm{SD})$ at 12 weeks postpartum and mean $0.64(0.56 \mathrm{SD})$ at 6 months postpartum. Correlations between these mPDI scores were high at all time-points postpartum: $0.72<$ rho $<0.86$. Agreement in parenting disability states at the different timepoints postpartum was moderate: $0.43<$ kappa $<0.60$.

\section{Identification of patients at risk for parenting difficulties}

The results of the univariate and multivariate logistic regression analyses for parenting disability at 12 weeks postpartum are shown in tables 2 and 3 . The observed median HAQ score in the first trimester of pregnancy was 0.625 . Multivariate logistic regression revealed that patients with a low HAQ score $(\leq 0.625)$ in the first trimester of pregnancy (OR 9.17, 95\% CI 3.04 to 27.69, p<0.001) and low disease activity in the first trimester (DAS28CRP <3.2) (OR 4.79, 95\% CI 1.77 to $12.91, \mathrm{p}=0.002$ ) were likely to report low parenting disability. Patients with a longer disease duration (OR $0.87,95 \%$ CI 0.79 to 0.95 , $p=0.003$, per year increase in disease activity) were less likely to report low parenting disability postpartum. Reporting a high HAQ score in the first trimester of pregnancy greatly increased the odds of reporting high parenting disability postpartum (OR 4.54, 95\% CI 1.99 to $10.34, \mathrm{p}<0.001)$. The presence of erosive disease was borderline significantly associated with reporting high parenting disability (OR $2.32,95 \%$ CI 1.00 to $5.35, \mathrm{p}=0.049$ ).

To confirm the results of the multivariate logistic regression and for clinical use, we stratified patients to having a high or low HAQ in the first trimester, after that these two groups were stratified to having low or high disease activity in the first trimester. The results are shown in table 4. Only $2.7 \%$ of the patients reported low parenting disability postpartum when having a high HAQ and high disease activity in the first trimester, and $86.5 \%$ of the patients in the low parenting disability group had a low HAQ score in the first trimester. On the contrary, only $8.3 \%$ of the patients that had both a low HAQ score and low disease activity in the first trimester reported high parenting disability postpartum. 


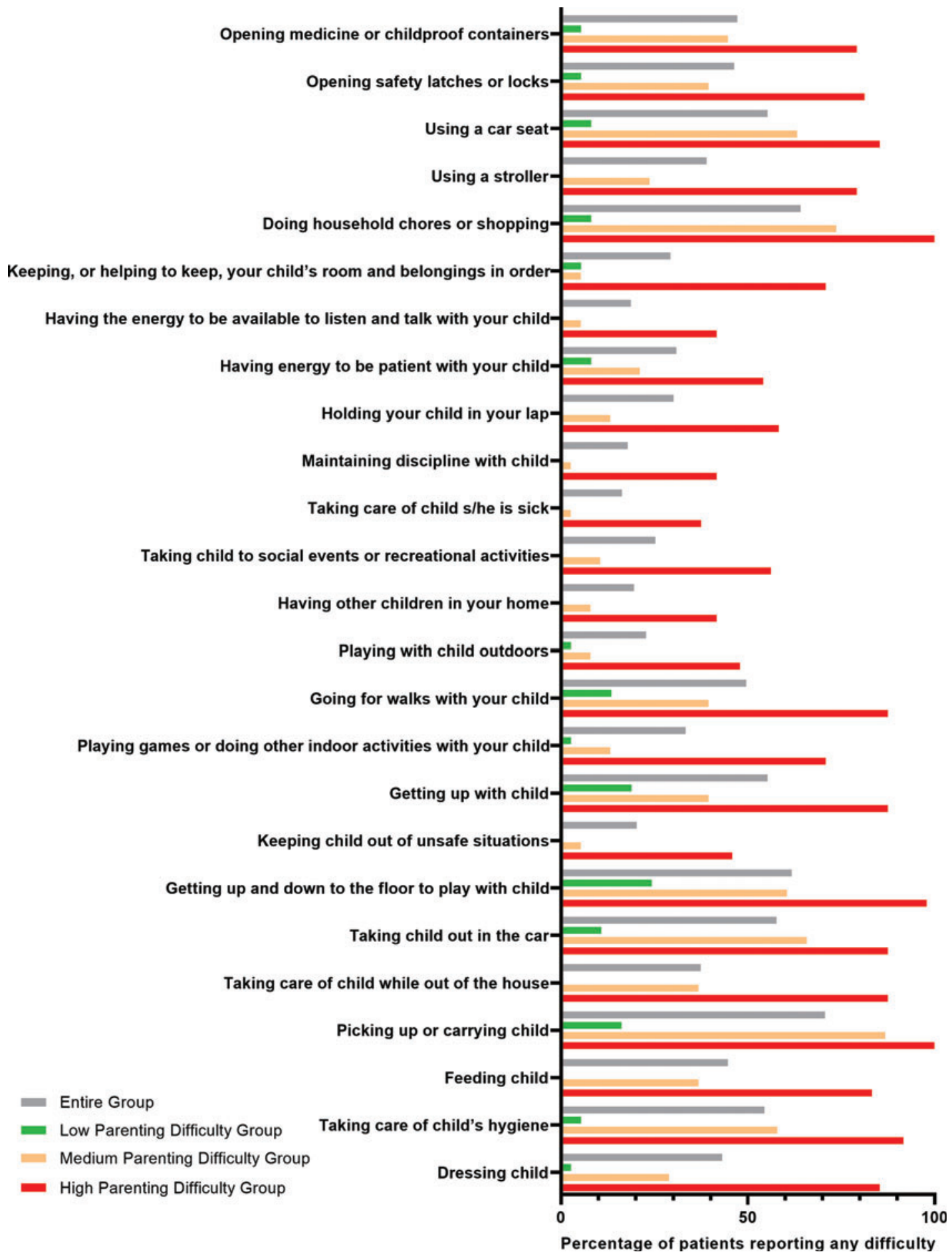

Figure 1 Histogram showing the percentage of patients (\%) that reported any difficulty for different domains of parenting. Results are shown for the entire group of patients and for the different parenting disability states at 12 weeks postpartum.

\section{DISCUSSION}

Our study was the first to prospectively evaluate the PDI in a large group of mothers with RA. It provided detailed information for patients and healthcare professionals on parenting postpartum. We identified that in patients with RA postpartum, physical domains of parenting were most commonly affected: picking up and carrying, hygiene needs, feeding, getting up and down, household chores, shopping and getting out in the car. Domains with a social/psychosocial component caused less problems: maintaining discipline, taking care of the children when they are sick, listening and having other children over. We identified that patients with a low HAQ score in the first trimester of pregnancy and low disease activity in the first trimester were likely to report low parenting disability. Patients with a longer disease duration were less likely 
Table 2 Patient characteristics (obtained at first trimester of pregnancy) associated with parenting difficulty at 12 weeks postpartum $(n=123)$ : multivariate logistic regression model for low parenting disability

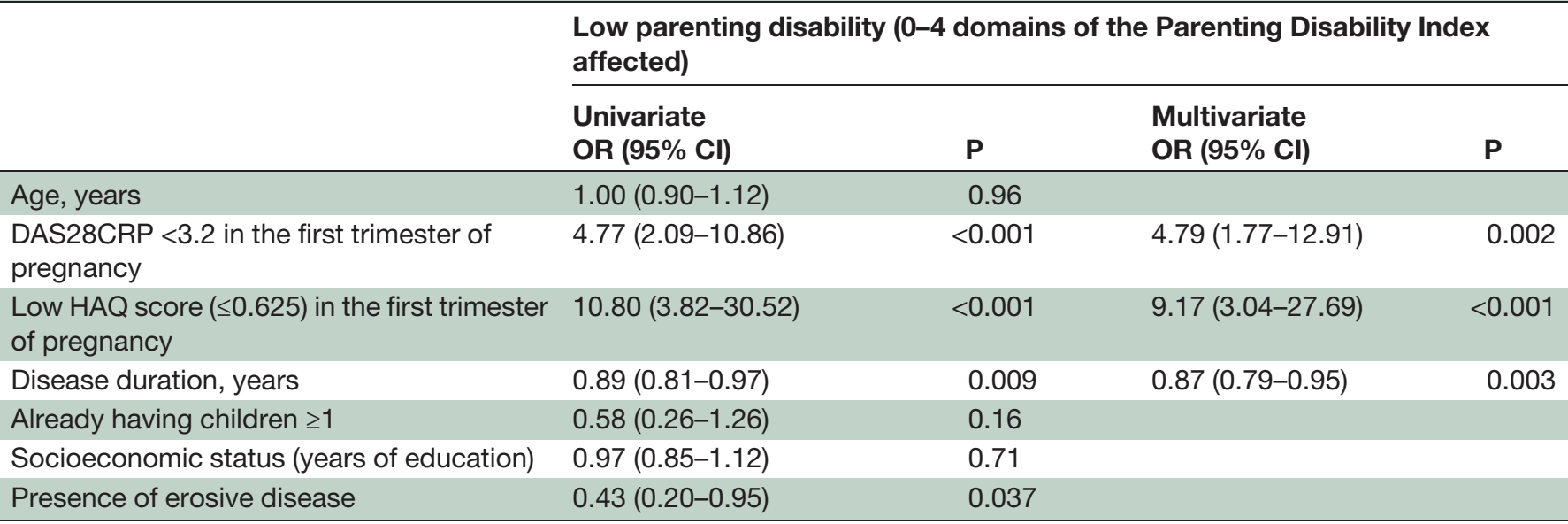

DAS28CRP, Disease Activity Score 28-joint count C reactive protein; HAQ, Health Assessment Questionnaire.

Table 3 Patient characteristics (obtained at first trimester of pregnancy) associated with parenting difficulty at 12 weeks postpartum ( $n=123)$ : multivariate logistic regression model for high parenting disability

\begin{tabular}{|c|c|c|c|c|}
\hline & \multicolumn{4}{|c|}{$\begin{array}{l}\text { High parenting disability (13-25 domains of the Parenting Disability Index } \\
\text { affected) }\end{array}$} \\
\hline & $\begin{array}{l}\text { Univariate } \\
\text { OR }(95 \% \mathrm{Cl})\end{array}$ & $\mathbf{P}$ & $\begin{array}{l}\text { Multivariate } \\
\text { OR }(95 \% \mathrm{Cl})\end{array}$ & $\mathbf{P}$ \\
\hline Age, years & $1.05(0.95-1.17)$ & 0.99 & & \\
\hline $\begin{array}{l}\text { DAS28CRP } \geq 3.2 \text { in the } 1 \text { st trimester of } \\
\text { pregnancy }\end{array}$ & $1.50(1.08-2.09)$ & 0.015 & $1.68(0.71-3.96)$ & 0.23 \\
\hline $\begin{array}{l}\text { High HAQ score }(>0.625) \text { in the 1st trimester } \\
\text { of pregnancy }\end{array}$ & $4.86(2.21-10.66)$ & $<0.001$ & $4.54(1.99-10.34)$ & $<0.001$ \\
\hline Disease duration, years & $1.05(0.99-1.12)$ & 0.11 & & \\
\hline Already having children $\geq 1$ & $1.44(0.69-2.99)$ & 0.33 & & \\
\hline Social economic status (years of education) & $0.90(0.79-1.03)$ & 0.12 & & \\
\hline Presence of erosive disease & $2.13(0.98-4.59)$ & 0.055 & $2.32(1.00-5.35)$ & 0.049 \\
\hline
\end{tabular}

DAS28CRP, Disease Activity Score 28-joint count C reactive protein; HAQ, Health Assessment Questionnaire.

Table 4 Stratification of patients for parenting disability states at 12 weeks postpartum by low or high HAQ score and low or high disease activity in first trimester of pregnancy $(n, \%)$

\begin{tabular}{|c|c|c|c|c|}
\hline & & $\begin{array}{l}\text { Low parenting } \\
\text { disability }\end{array}$ & $\begin{array}{l}\text { Medium parenting } \\
\text { disability }\end{array}$ & $\begin{array}{l}\text { High parenting } \\
\text { disability }\end{array}$ \\
\hline \multirow[t]{3}{*}{$\begin{array}{l}\text { High HAQ score in the first trimester } \\
(>0.625)\end{array}$} & $\begin{array}{l}\text { DAS28CRP } \\
\geq 3.2\end{array}$ & $1(2.7)$ & $17(44.7)$ & $25(52.1)$ \\
\hline & $\begin{array}{l}\text { DAS28CRP } \\
<3.2\end{array}$ & 4 (10.8) & 4 (10.5) & 9 (18.8) \\
\hline & Subtotal & 5 (13.5) & 28 (55.3) & $34(70.8)$ \\
\hline \multirow[t]{4}{*}{$\begin{array}{l}\text { Low HAQ score in the first trimester } \\
(\leq 0.625)\end{array}$} & $\begin{array}{l}\text { DAS28CRP } \\
\geq 3.2\end{array}$ & 12 (32.4) & $9(23.7)$ & $10(20.8)$ \\
\hline & $\begin{array}{l}\text { DAS28CRP } \\
<3.2\end{array}$ & $20(54.1)$ & 8 (21.1) & 4 (8.3) \\
\hline & Subtotal & 32 (86.5) & 17 (44.7) & 14 (29.2) \\
\hline & Total & 37 & 38 & 48 \\
\hline
\end{tabular}

DAS28CRP, Disease Activity Score 28-joint count C reactive protein; HAQ, Health Assessment Questionnaire. 
of reporting low parenting disability postpartum. Having a high HAQ score in the first trimester of pregnancy and the presence of erosive disease increased the risk for parenting disability postpartum.

Early identification of patients at risk for developing parenting problems postpartum will allow those patients extra time to take additional precautions. To assess the risk of developing parenting problems during counselling sessions, we advise physicians to take the following into account: disease activity, presence of erosive disease, disease duration and most importantly the HAQ score. In our analysis, we used the patient characteristics in the first trimester, because there were more patients included in our cohort at that time-point $(\mathrm{n}=116)$ than at the preconception visit $(\mathrm{n}=88)$. However, in some circumstances, counselling before pregnancy can be preferred. In our cohort, patients were seen before conception when contraindicated medication for use during pregnancy was stopped for at least 3 months. Pearson's coefficient between the HAQ in the first trimester and the HAQ before conception were good, rho $=0.74$. When we performed the multivariate logistic analysis with data obtained at the visit before conception, similar results were found (data not shown). A cut-off for the HAQ of 0.625 before conception or in the first trimester of pregnancy can be used to identify patients who are at risk for developing parenting problems, where a score of $>0.625$ indicates an increased risk of developing parenting problems.

With the identification of the parenting domains that cause the most difficulties for patients and which patients are at risk for developing parenting disability, targeted interventions and education can be designed. For patients with a low risk of developing parenting disability, an information folder with tips on positioning and carrying the baby, the advice to roll the baby over instead of lifting when changing and the advice to have a baby changing table on the ground floor could be sufficient. Whereas in the group with a high probability of developing parenting difficulty, a training by a specialised nurse or occupational therapist on positioning of the baby, taking care of the child's hygiene, the use of equipment (car seat, nursing pillow, stroller, etc) and strategies on adjusting expectations might be appropriate. Further research should be conducted among patients with and without the above-described intervention, in order to determine whether additional training results in better parenting function.

Considering our findings, it is tempting to speculate that interventions, focused on improving HAQ scores, can improve parenting. HAQ scores are influenced by a variety of lifestyle and disease- and treatment-related factors of which disease activity and prior radiologic damage are often considered most important. ${ }^{11}$ Although radiologic damage cannot be changed, effective drug therapy and lower disease activity can improve HAQ scores. ${ }^{12}$ Future research should reveal whether this can be achieved in patients with a wish to conceive or who are pregnant.
Our study has several limitations. The PARA study was completed around a decade ago, the patients population might therefore not reflect the current patient population. Differences can be expected in medication use and disease activity. In the time period that the PARA study was conducted treating RA during pregnancy was uncommon. Previous literature with data from this cohort shows that a large percentage of patients were not treated during pregnancy or were treated with sulfasalazine or prednisone. ${ }^{6}$ However, the conclusion that HAQ identifies patients with future parenting disability is still valid.

Also, the PDI questionnaire was not tested in a reference group of mothers without a chronic rheumatic disease. Therefore, the results of this questionnaire cannot be compared to mothers in the general population. Also, we did not validate the results of the multivariate logistic regression analysis in a reference cohort.

A flaw in the PDI questionnaire is that it does not include questions on whether a patient received additional help with parenting. Additional help from, for example, a partner, (grand) parents or maternity assistant can make parenting tasks easier. We asked participants the number of days on which they received help from any third-party after delivery. This number of days did not differ between women who experienced high parenting disability postpartum versus women who did not (data not shown). Although this is only a proxy for the total amount of help received, it indicates that both groups received a similar amount of help in parenting tasks.

The current study was performed in a country where all patients are entitled to maternal leave and have easy access to healthcare. In other countries, a chronic disease might have a greater impact on parenting capability. Therefore, our results cannot be extrapolated. We do not suspect selection bias in the current study, because the number of missing values in our study is low and previous published literature from the PARA study showed that this study is a representative study population.

We observed no major differences in PDI score at different follow-up visits postpartum. This is probably explained by only slight changes in disease activity. The highest PDI score was observed at 12 weeks after delivery, which corresponds with the timing of a flare of disease activity postpartum found in literature. ${ }^{6}$

Our study reports slightly lower mPDI scores than the initial validation study by Katz et $a l$ and a more recent study by Zelkowitz et al. ${ }^{513}$ These differences can probably be explained by cultural differences between countries and a different study design.

In conclusion, physical domains of parenting are most commonly affected in mothers with RA. The domains picking up and carrying children, household chores, taking children out in the car, getting up and down to the floor to play and child's hygiene are affected in a large percentage of patients with RA. Therefore, during pregnancy counselling sessions, advice and 
instructions on these domains should be provided to all pregnant patients with RA, since postpartum parenting disability was influenced by disease activity. We advise that modifiable factors, such as effective drug therapy, should be discussed during counselling sessions to preserve the parenting tasks. Some patients might need more in-depth counselling, these patients can be identified in early pregnancy based upon high DAS28CRP and especially high HAQ scores.

Contributors All authors had a contribution to the conception and/or design of the manuscript and/or the acquisition/analysis/interpretation of the data. They revised the manuscript critically and gave final approval. HTWS and RJEMD take full responsibility for all aspects regarding the accuracy and integrity of the work.

Funding This work was supported by the Dutch Arthritis Foundation (ReumaNederland) (project number: LLP-26). RJEMD received an unrestricted grant from UCB Pharma.

Competing interests RJEMD received an unrestricted grant from UCB Pharma.

Patient consent for publication Not required.

Provenance and peer review Not commissioned; externally peer reviewed.

Data availability statement Data may be obtained from a third party and are not publicly available.

Open access This is an open access article distributed in accordance with the Creative Commons Attribution Non Commercial (CC BY-NC 4.0) license, which permits others to distribute, remix, adapt, build upon this work non-

commercially, and license their derivative works on different terms, provided the original work is properly cited, appropriate credit is given, any changes made indicated, and the use is non-commercial. See: http://creativecommons. org/licenses/by-nc/4.0/.

ORCID iDs

Hieronymus T W Smeele http://orcid.org/0000-0001-7724-7712

\section{REFERENCES}

1 Barlow JH, Cullen LA, Foster NE, et al. Does arthritis influence perceived ability to fulfill a parenting role? Perceptions of mothers, fathers and grandparents. Patient Educ Couns 1999;37:141-51.

2 Phillips R, Pell B, Grant A, et al. Identifying the unmet information and support needs of women with autoimmune rheumatic diseases during pregnancy planning, pregnancy and early parenting: mixed-methods study. BMC Rheumatol 2018;2:21.

3 Chew C, Rebić N, Baldwin C, et al. "r/Thritis", pregnancy, and parenting: a qualitative descriptive study of reddit forums to explore information needs and concerns of women with rheumatoid arthritis. ACR Open Rheumatol 2019;1:485-92.

4 Ostensen M. Contraception and pregnancy counselling in rheumatoid arthritis. Curr Opin Rheumatol 2014;26:302-7.

5 Katz PP, Pasch LA, Wong B. Development of an instrument to measure disability in parenting activity among women with rheumatoid arthritis. Arthritis Rheum 2003;48:935-43.

6 de Man YA, Dolhain RJ, van de Geijn FE, et al. Disease activity of rheumatoid arthritis during pregnancy: results from a nationwide prospective study. Arthritis Rheum 2008;59:1241-8.

7 Arnett FC, Edworthy SM, Bloch DA, et al. The American Rheumatism Association 1987 revised criteria for the classification of rheumatoid arthritis. Arthritis Rheum 1988;31:315-24.

8 Siegert CE, Vleming LJ, Vandenbroucke JP, et al. Measurement of disability in Dutch rheumatoid arthritis patients. Clin Rheumatol 1984;3:305-9.

9 McHugh ML. Interrater reliability: the kappa statistic. Biochem Med (Zagreb) 2012;22:276-82.

10 Schober P, Boer C, Schwarte LA. Correlation coefficients: appropriate use and interpretation. Anesth Analg 2018;126:1763-8.

11 Drossaers-Bakker KW, De Buck M, Van Zeben D, et al. Long-term course and outcome of functional capacity in rheumatoid arthritis: the effect of disease activity and radiologic damage over time. Arthritis Rheum 1999;42:1854-60.

12 Barra L, Ha A, Sun L, et al. Efficacy of biologic agents in improving the Health Assessment Questionnaire $(\mathrm{HAQ})$ score in established and early rheumatoid arthritis: a meta-analysis with indirect comparisons. Clin Exp Rheumatol 2014;32:333-41.

13 Zelkowitz P, Looper KJ, Mustafa SS, et al. McGill early arthritis research G. Parenting disability, parenting stress and child behaviour in early inflammatory arthritis. Chronic Dis Inj Can 2013;33:81-7. 